Network Working Group

Request for Comments: 1196

Obsoletes: RFCs 1194, 742
D. Zimmerman

Center for Discrete Mathematics and Theoretical Computer Science

December 1990

The Finger User Information Protocol

Status of this Memo

This memo defines a protocol for the exchange of user information. This RFC specifies an IAB standards track protocol for the Internet community, and requests discussion and suggestions for improvements. Please refer to the current edition of the "IAB Official Protocol Standards" for the standardization state and status of this protocol. Distribution of this memo is unlimited.

Abstract

This memo describes the Finger User Information Protocol. This is a simple protocol which provides an interface to a remote user information program.

Based on RFC 742, a description of the original Finger protocol, this memo attempts to clarify the expected communication between the two ends of a Finger connection. It also tries not to invalidate the many existing implementations or add unnecessary restrictions to the original protocol definition. This edition corrects and clarifies in a minor way, RFC 1194 .

Table of Contents

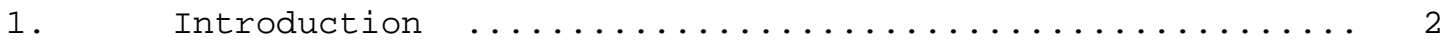

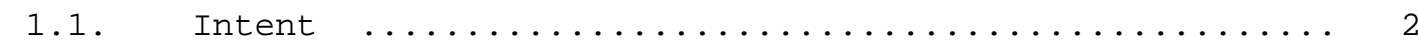

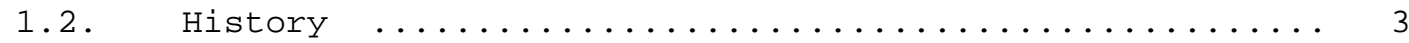

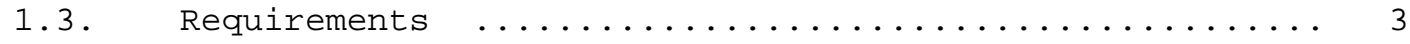

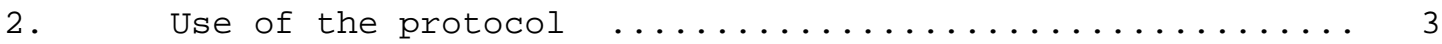

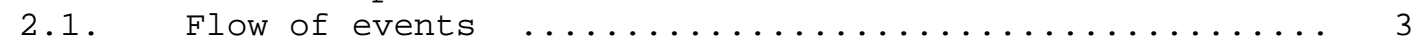

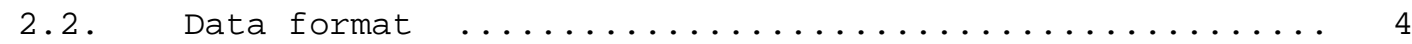

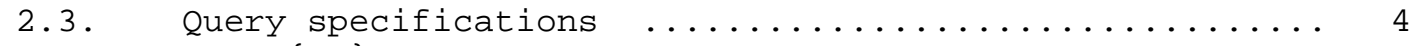

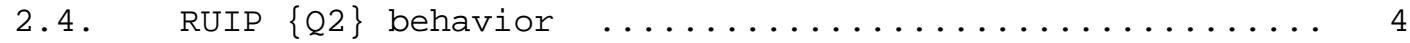

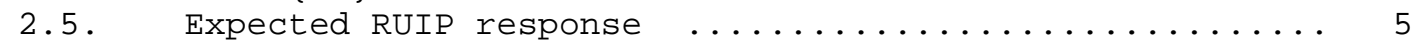

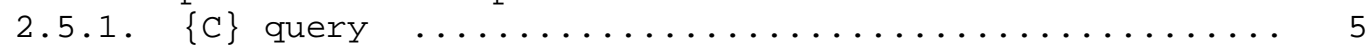

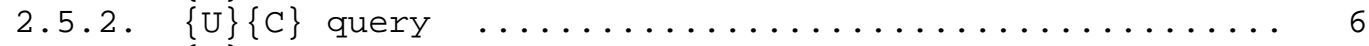

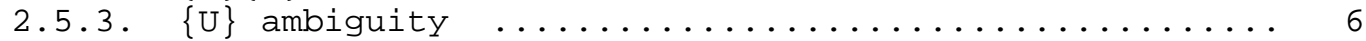

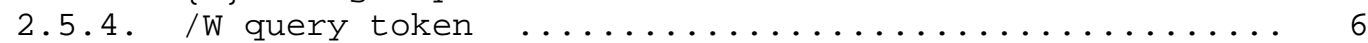

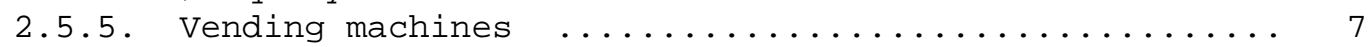

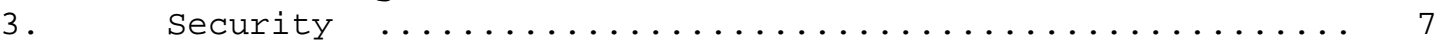




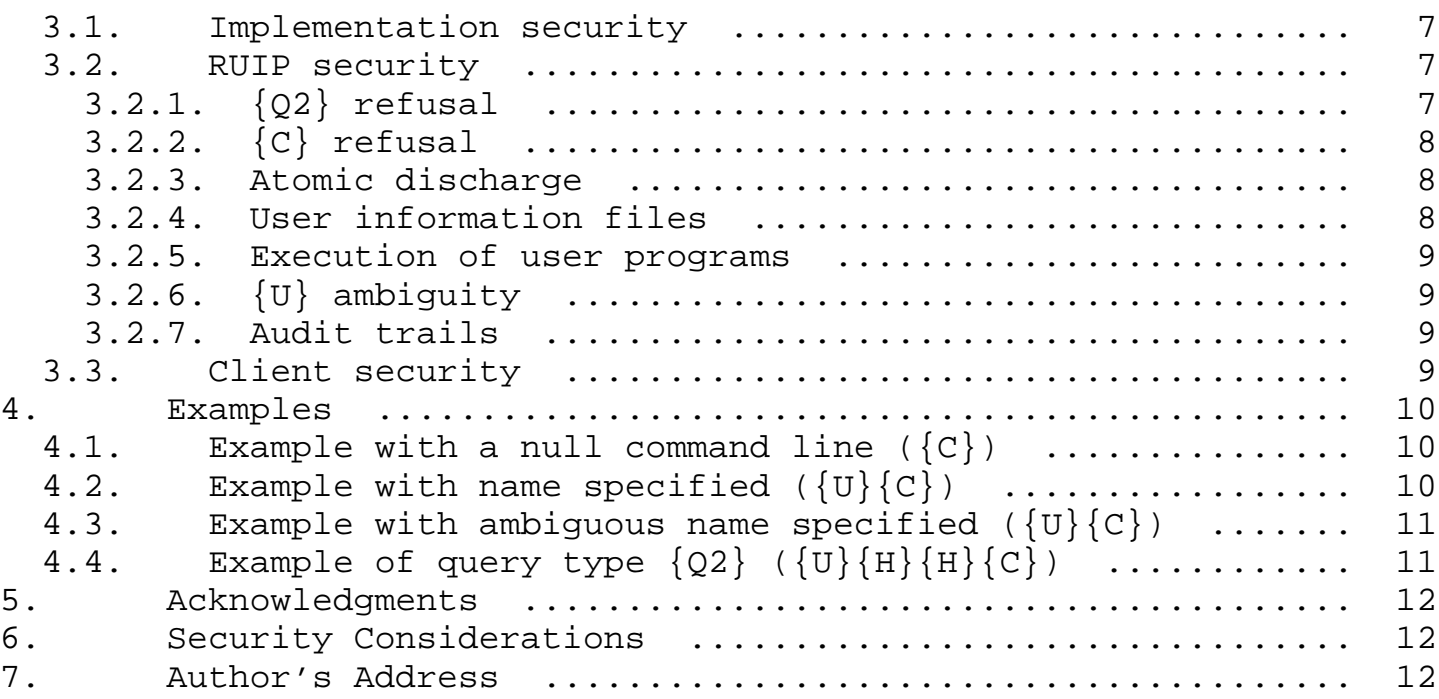

1. Introduction

\subsection{Intent}

This memo describes the Finger User Information Protocol. This is a simple protocol which provides an interface to a remote user information program (RUIP).

Based on RFC 742, a description of the original Finger protocol, this memo attempts to clarify the expected communication between the two ends of a Finger connection. It also tries not to invalidate the many current implementations or add unnecessary restrictions to the original protocol definition.

The most prevalent implementations of Finger today seem to be primarily derived from the BSD UNIX work at the University of California, Berkeley. Thus, this memo is based around the BSD version's behavior.

However, the BSD version provides few options to tailor the Finger RUIP for a particular site's security policy, or to protect the user from dangerous data. Furthermore, there are MANY potential security holes that implementors and administrators need to be aware of, particularly since the purpose of this protocol is to return information about a system's users, a sensitive issue at best. Therefore, this memo makes a number of important security comments and recommendations. 


\subsection{History}

The FINGER program at SAIL, written by Les Earnest, was the inspiration for the NAME program on ITS. Earl Killian at MIT and Brian Harvey at SAIL were jointly responsible for implementing the original protocol.

Ken Harrenstien is the author of RFC 742, "Name/Finger", which this memo began life as.

\subsection{Requirements}

In this document, the words that are used to define the significance of each particular requirement are capitalized. These words are:

* "MUST"

This word or the adjective "REQUIRED" means that the item is an absolute requirement of the specification.

* "SHOULD"

This word or the adjective "RECOMMENDED" means that there may exist valid reasons in particular circumstances to ignore this item, but the full implications should be understood and the case carefully weighed before choosing a different course.

* "MAY"

This word or the adjective "OPTIONAL" means that this item is truly optional. One vendor may choose to include the item because a particular marketplace requires it or because it enhances the product, for example; another vendor may omit the same item.

An implementation is not compliant if it fails to satisfy one or more of the MUST requirements. An implementation that satisfies all the MUST and all the SHOULD requirements is said to be "unconditionally compliant"; one that satisfies all the MUST requirements but not all the SHOULD requirements is said to be "conditionally compliant".

2. Use of the protocol

\subsection{Flow of events}

Finger is based on the Transmission Control Protocol, using TCP port 79 decimal (117 octal). A TCP connection is opened to a remote host on the Finger port. An RUIP becomes available on the remote end of the connection to process the request. The RUIP is sent a one line 
query based upon the Finger query specification. The RUIP processes the query, returns an answer, then closes the connection normally.

\subsection{Data format}

Any data transferred MUST be in ASCII format, with no parity, and with lines ending in CRLF (ASCII 13 followed by ASCII 10). This excludes other character formats such as EBCDIC, etc. This also means that any characters between ASCII 128 and ASCII 255 should truly be international data, not 7-bit ASCII with the parity bit set.

\subsection{Query specifications}

An RUIP MUST accept the entire Finger query specification.

The Finger query specification is defined:

$$
\begin{array}{ll}
\{\mathrm{Q} 1\} & ::=[\{\mathrm{U}\}][/ \mathrm{W}] \quad\{\mathrm{C}\} \\
\{\mathrm{Q} 2\} & ::=[\{\mathrm{U}\}]\{\mathrm{H}\}[/ \mathrm{W}] \quad\{\mathrm{C}\} \\
\{\mathrm{U}\} & ::=\text { username } \\
\{\mathrm{H}\} & ::=\text { ehostname } \mid \text { ehostname }\{\mathrm{H}\} \\
\{\mathrm{C}\} & ::=<\mathrm{CRLF}>
\end{array}
$$

$\{\mathrm{H}\}$, being recursive, means that there is no arbitrary limit on the number of chostname tokens in the query. In examples of the $\{\mathrm{Q} 2\}$ request specification, the number of dhostname tokens is limited to two, simply for brevity.

Be aware that $\{Q 1\}$ and $\{Q 2\}$ do not refer to a user typing "finger user@host" from an operating system prompt. It refers to the line that an RUIP actually receives. So, if a user types "finger userahost $\angle \mathrm{CRLF}>$ ", the RUIP on the remote host receives "user $<$ CRLF $>$ ", which corresponds to $\{\mathrm{Q} 1\}$.

As with anything in the IP protocol suite, "be liberal in what you accept".

\section{4. $\operatorname{RUIP}\{\mathrm{Q} 2\}$ behavior}

A query of $\{\mathrm{Q} 2\}$ is a request to forward a query to another RUIP. An RUIP MUST either provide or actively refuse this forwarding service (see section 3.2.1). If an RUIP provides this service, it MUST conform to the following behavior: 
Given that:

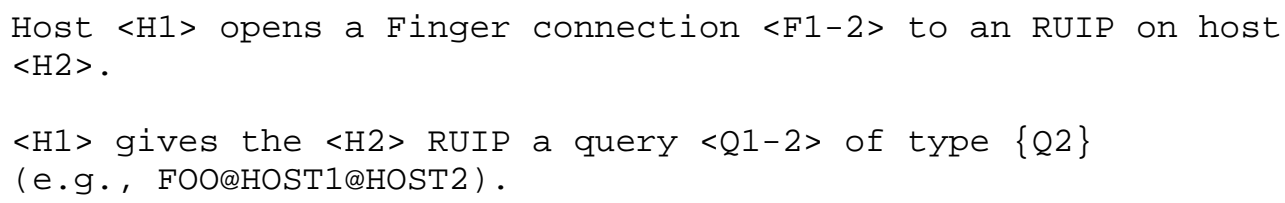

And so:

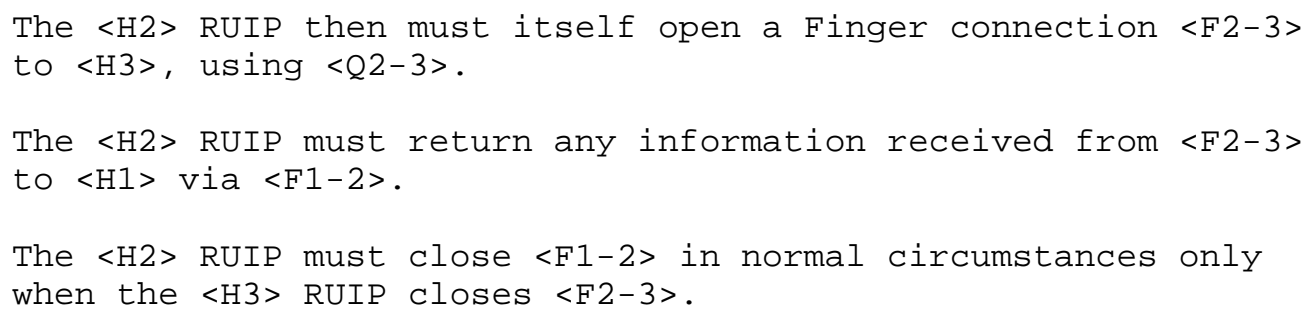

2.5. Expected RUIP response

For the most part, the output of an RUIP doesn't follow a strict specification, since it is designed to be read by people instead of programs. It should mainly strive to be informative.

Output of ANY query is subject to the discussion in the security section.

\subsection{1. $\{\mathrm{C}\}$ query}

A query of $\{C\}$ is a request for a list of all online users. An RUIP MUST either answer or actively refuse (see section 3.2.2). If it answers, then it MUST provide at least the user's full name. The system administrator SHOULD be allowed to include other useful information (per section 3.2.3), such as:

- terminal location

- office location

- office phone number

- job name

- idle time (number of minutes since last typed input, or 
since last job activity).

\subsection{2. $\{\mathrm{U}\}\{\mathrm{C}\}$ query}

A query of $\{U\}\{C\}$ is a request for in-depth status of a specified user $\{U\}$. If you really want to refuse this service, you probably don't want to be running Finger in the first place.

An answer MUST include at least the full name of the user. If the user is logged in, at least the same amount of information returned by $\{C\}$ for that user MUST also be returned by $\{U\}\{C\}$.

Since this is a query for information on a specific user, the system administrator SHOULD be allowed to choose to return additional useful information (per section 3.2.3), such as:

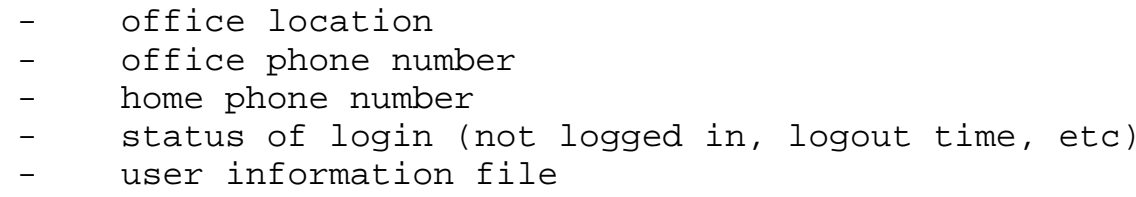

A user information file is a feature wherein a user may leave a short message that will be included in the response to Finger requests. (This is sometimes called a "plan" file.) This is easily implemented by (for example) having the program look for a specially named text file in the user's home directory or some common area; the exact method is left to the implementor. The system administrator SHOULD be allowed to specifically turn this feature on and off. See section 3.2.4 for caveats.

There MAY be a way for the user to run a program in response to a Finger query. If this feature exists, the system administrator SHOULD be allowed to specifically turn it on and off. See section 3.2 .5 for caveats.

\subsection{3. $\{\mathrm{U}\}$ ambiguity}

Allowable "names" in the command line MUST include "user names" or "login names" as defined by the system. If a name is ambiguous, the system administrator SHOULD be allowed to choose whether or not all possible derivations should be returned in some fashion (per section $3.2 .6)$.

\subsection{4. /W query token}

The token $/ W$ in the $\{Q 1\}$ or $\{Q 2\}$ query types SHOULD at best be interpreted at the last RUIP to signify a higher level of verbosity 
in the user information output, or at worst be ignored.

\subsubsection{Vending machines}

Vending machines SHOULD respond to a $\{\mathrm{C}\}$ request with a list of all items currently available for purchase and possible consumption. Vending machines SHOULD respond to a $\{U\}\{C\}$ request with a detailed count or list of the particular product or product slot. Vending machines should NEVER NEVER EVER eat requests. Or money.

3. Security

\subsection{Implementation security}

Sound implementation of Finger is of the utmost importance. Implementations should be tested against various forms of attack. In particular, an RUIP SHOULD protect itself against malformed inputs. Vendors providing Finger with the operating system or network software should subject their implementations to penetration testing.

Finger is one of the avenues for direct penetration, as the Morris worm pointed out quite vividly. Like Telnet, FTP and SMTP, Finger is one of the protocols at the security perimeter of a host. Accordingly, the soundness of the implementation is paramount. The implementation should receive just as much security scrutiny during design, implementation, and testing as Telnet, FTP, or SMTP.

\subsection{RUIP security}

Warning!! Finger discloses information about users; moreover, such information may be considered sensitive. Security administrators should make explicit decisions about whether to run Finger and what information should be provided in responses. One existing implementation provides the time the user last logged in, the time he last read mail, whether unread mail was waiting for him, and who the most recent unread mail was from! This makes it possible to track conversations in progress and see where someone's attention was focused. Sites that are information-security conscious should not run Finger without an explicit understanding of how much information it is giving away.

\subsection{1. $\{Q 2\}$ refusal}

For individual site security concerns, the system administrator SHOULD be given an option to individually turn on or off RUIP processing of $\{Q 2\}$. If RUIP processing of $\{Q 2\}$ is turned off, the RUIP MUST return a service refusal message of some sort. "Finger forwarding service denied" is adequate. The purpose of this is to 
allow individual hosts to choose to not forward Finger requests, but if they do choose to, to do so consistently.

Overall, there are few cases which would warrant processing of $\{Q 2\}$ at all, and they are far outweighed by the number of cases for refusing to process $\{02\}$. In particular, be aware that if a machine is part of security perimeter (that is, it is a gateway from the outside world to some set of interior machines), then turning $\{Q 2\}$ on provides a path through that security perimeter. Therefore, it is RECOMMENDED that the default of the $\{Q 2\}$ processing option be to refuse processing. It certainly should not be enabled in gateway machines without careful consideration of the security implications.

\subsection{2. $\{$ C $\}$ refusal}

For individual site security concerns, the system administrator SHOULD be given an option to individually turn on or off RUIP acceptance of $\{C\}$. If RUIP processing of $\{C\}$ is turned off, the RUIP MUST return a service refusal message of some sort. "Finger online user list denied" is adequate. The purpose of this is to allow individual hosts to choose to not list the users currently online.

\subsubsection{Atomic discharge}

All implementations of Finger SHOULD allow individual system administrators to tailor what atoms of information are returned to a query. For example:

- Administrator A should be allowed to specifically choose to return office location, office phone number, home phone number, and logged in/logout time.

- Administrator B should be allowed to specifically choose to return only office location, and office phone number.

- Administrator $\mathrm{C}$ should be allowed to specifically choose to return the minimum amount of required information, which is the person's full name.

\subsubsection{User information files}

Allowing an RUIP to return information out of a user-modifiable file should be seen as equivalent to allowing any information about your system to be freely distributed. That is, it is potentially the same as turning on all specifiable options. This information security breach can be done in a number of ways, some cleverly, others straightforwardly. This should disturb the sleep of system administrators who wish to control the returned information. 


\subsubsection{Execution of user programs}

Allowing an RUIP to run a user program in response to a Finger query is potentially dangerous. BE CAREFUL!! -- the RUIP MUST NOT allow system security to be compromised by that program. Implementing this feature may be more trouble than it is worth, since there are always bugs in operating systems, which could be exploited via this type of mechanism.

\subsection{6. $\{\mathrm{U}\}$ ambiguity}

Be aware that a malicious user's clever and/or persistent use of this feature can result in a list of most of the usernames on a system. Refusal of $\{U\}$ ambiguity should be considered in the same vein as refusal of $\{C\}$ requests (see section 3.2.2).

\subsubsection{Audit trails}

Implementations SHOULD allow system administrators to log Finger queries.

\subsection{Client security}

It is expected that there will normally be some client program that the user runs to query the initial RUIP. By default, this program SHOULD filter any unprintable data, leaving only printable 7-bit characters (ASCII 32 through ASCII 126), tabs (ASCII 9), and CRLFs. This is to protect against people playing with terminal escape codes, changing other peoples' $\mathrm{X}$ window names, or committing other dastardly or confusing deeds. Two separate user options SHOULD be considered to modify this behavior, so that users may choose to view international or control characters:

- one to allow all characters less than ASCII 32

- another to allow all characters greater than ASCII 126

For environments that live and breathe international data, the system administrator SHOULD be given a mechanism to enable the latter option by default for all users on a particular system. This can be done via a global environment variable or similar mechanism. 


\section{Examples}

4.1. Example with a null command line ( $\{\mathrm{C}\}$ )

Site: elbereth.rutgers.edu

Command line: <CRLF>

\begin{tabular}{|c|c|c|c|c|c|c|}
\hline Login & Name & TTY & Idle & When & \multicolumn{2}{|c|}{ Office } \\
\hline rinehart & Mark J. Rinehart & p0 & $1: 11$ & Mon $12: 15$ & $019 \mathrm{Hill}$ & $\times 3166$ \\
\hline enfie & Stephen J. Greenfiel & $\mathrm{p} 1$ & & Mon 15:46 & $542 \mathrm{Hill}$ & 74 \\
\hline atel & Rocky - Rakesh Patel & p3 & $4 d$ & Thu $00: 58$ & $028 \mathrm{Hill}$ & 287 \\
\hline sant & Mel Pleasant & $\mathrm{p} 4$ & $3 d$ & Thu $21: 32$ & 19 Hill & $32-$ \\
\hline ip & Dave Phillips & p5 & $021:$ & Sun $18: 24$ & $265 \mathrm{Hill}$ & 92 \\
\hline & David Katinsky & $\mathrm{p} 6$ & $2 \mathrm{~d}$ & Thu 14:11 & $028 \mathrm{Hill}$ & $x 2$ \\
\hline iss & Cary Cherniss & $\mathrm{p} 7$ & 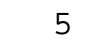 & Mon $15: 42$ & 127 Psycho & $x 2$ \\
\hline ga & Doug Harnaga & $\mathrm{p} 8$ & $2: 01$ & Mon 10:15 & $055 \mathrm{Hill}$ & $x 2351$ \\
\hline & Thomas P. Brisco & pe & $2: 09$ & Mon 13:37 & 1055 & $x 2351$ \\
\hline & Angus Laidlaw & q0 & $1: 55$ & Mon 11:26 & E313C & $8-5$ \\
\hline & Chris Jarocha-Ernst & q1 & 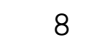 & Mon $13: 43$ & $259 \mathrm{Hill}$ & $x 2413$ \\
\hline
\end{tabular}

4.2. Example with name specified ( $\{\mathrm{U}\}\{\mathrm{C}\}$ )

Site: dimacs.rutgers.edu

Command line: pirmann<CRLF>

Login name: pirmann

Office: $016 \mathrm{Hill}$, x2443

Directory: /dimacs/u1/pirmann

In real life: David Pirmann

No unread mail

Project :

Plan:

Work Schedule, Summer 1990

Rutgers LCSR Operations, 908-932-2443

$\begin{array}{ll}\text { Monday } & 5 \mathrm{pm}-12 \mathrm{am} \\ \text { Tuesday } & 5 \mathrm{pm}-12 \mathrm{am} \\ \text { Wednesday } & 9 \mathrm{am}-5 \mathrm{pm} \\ \text { Thursday } & 9 \mathrm{am}-5 \mathrm{pm} \\ \text { Saturday } & 9 \mathrm{am}-5 \mathrm{pm} \\ \quad & \\ \quad \text { larf larf hoo hoo }\end{array}$

Zimmerman

[Page 10] 


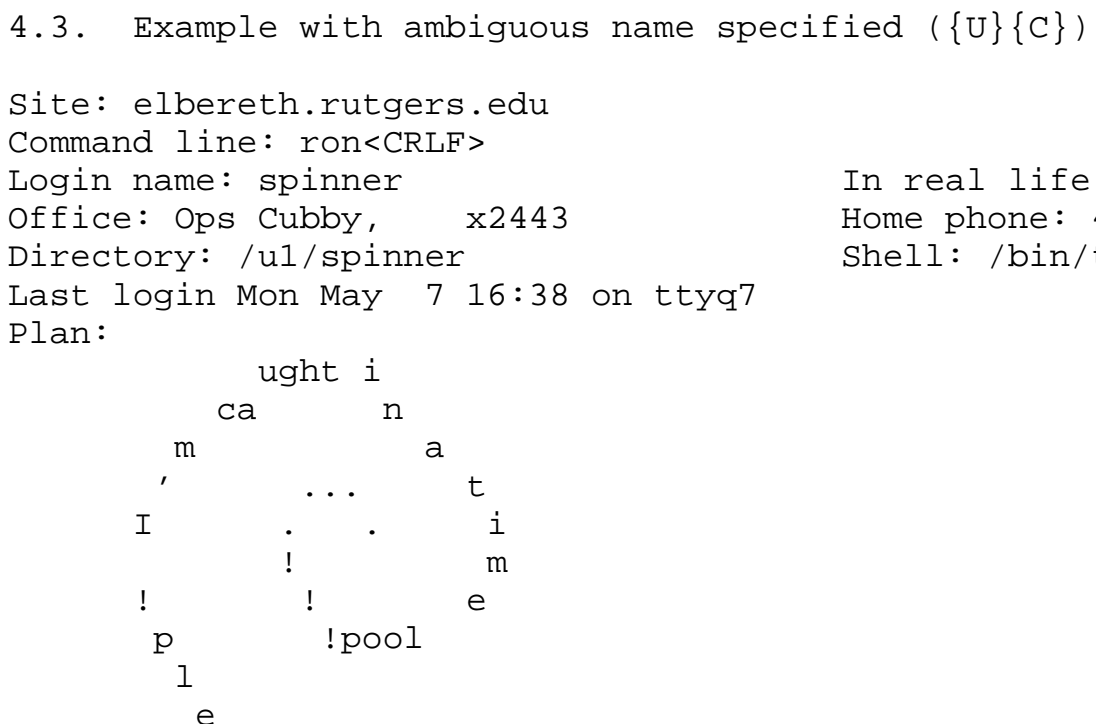


5. Acknowledgments

Thanks to everyone in the Internet Engineering Task Force for their comments. Special thanks to Steve Crocker for his security recommendations and prose.

6. Security Considerations

Security issues are discussed in section 3.

7. Author's Address

David Paul Zimmerman

Center for Discrete Mathematics and

Theoretical Computer Science

Rutgers University

P.O. Box 1179

Piscataway, NJ 08855-1179

Phone: (908) 932-4592

EMail: dpz@dimacs.rutgers.edu 